\title{
Rapid inactivation in vitro of SARS-CoV-2 in saliva by black tea and green tea
}

\author{
Eriko Ohgitani $^{1 *}$, Masaharu Shin-Ya ${ }^{1 *}$, Masaki Ichitani ${ }^{2}$, Makoto \\ Kobayashi ${ }^{2}$, Takanobu Takihara ${ }^{2}$, Masaya Kawamoto ${ }^{1}$, Hitoshi \\ Kinugasa $^{2}$ and Osam Mazda ${ }^{1 \#}$ \\ ${ }^{1}$ Department of Immunology, Kyoto Prefectural University of Medicine, Kamigyo, \\ Kyoto 602-8566, Japan. \\ ${ }^{2}$ Central Research Institute, ITO EN, Ltd., Makinohara, Shizuoka 421-0516, \\ Japan \\ *These authors equally contributed to this study. \\ \#Corresponding author.
}




\section{Abstract}

Saliva plays major roles in human-to-human transmission of the SARS-CoV-2. Recently we reported that black, green and oolong tea significantly inactivated SARS-CoV-2 within $1 \mathrm{~min}$. Theaflavin-3,3'-di-gallate (TFDG), theasinensin A (TSA) and (-) epigallocatechin gallate (EGCG) were involved in the anti-viral activities. Here we examined how long period is required for the compounds to inactivate the virus. We also assessed whether tea inactivates SARS-CoV-2 diluted in human saliva. Treatment of SARS-CoV-2 with $500 \mu \mathrm{M}$ TFDG or TSA for $10 \mathrm{sec}$ reduced the virus titer to undetectable levels (less than 1/1,000). Black and green tea decreased virus titer to less than $1 / 100$ within $10 \mathrm{sec}$ even in saliva. These findings suggest a possibility that intake of, or gargling with, tea may inactivate SARS-CoV-2 in saliva in infected individuals, which may eventually attenuate spread of COVID-19 within a population, although clinical studies are required to test this hypothesis by determining the intensity and duration of the anti-viral effect of tea in saliva in humans.

Keywords: Novel coronavirus, SARS-CoV-2, COVID-19, Tea, Tea catechin, Theaflavin, Polyphenols, Flavan-3-ols, saliva 
Introduction

The pandemic of novel coronavirus SARS-CoV-2 is expanding across the world with more than 80 million confirmed cases, and global death toll from COVID-19 has exceeded 1.7 million. Prophylactic procedures to attenuate the spread of virus infection is urgently needed.

SARS-CoV-2 is transmitted from a person to another principally through droplet infection, contact infection and aerosol infection ${ }^{1}$. SARS-CoV-2 in saliva, sputum or nasal discharge is released from patients or infected individuals by talking, sneezing, coughing, and singing, and reaches nasopharyngeal, oral, or conjunctival mucosa of nearby persons through inhalation, ingestion, touching by fingers, etc ${ }^{2-6}$. Especially, virus in saliva plays important roles, because saliva is commonly excreted during talking that is essential for our ordinary communication to each other as well as for economic and social activities in our societies. If the SARS-CoV-2 in saliva of infected individuals is effectively inactivated by a procedure, such a procedure could be useful for attenuating spread of the virus infection within a population.

We recently found that SARS-CoV-2 is significantly inactivated by a treatment with green tea, roasted green tea, oolong tea, and most remarkably, black tea ${ }^{7}$. One min was sufficient for the inactivation of the virus by tea. As the active constituents, we found that (-) epigallocatechin gallate (EGCG) and theaflavin-3,3'-di-gallate (TFDG) that are bioactive compounds in green and black tea, respectively, and theasinensin A (TAS) that is a dimer of EGCG strongly inactivated SARS-CoV-2. These compounds significantly inhibited interaction between recombinant ACE2 and RBD of the Spike proteins, which may be, at least partially, involved in the mechanism underlying the anti-coronavirus effect of tea.

Therefore, tea could be used to inactivate SARS-CoV-2 in saliva in infected persons simply by ingesting or gargling with it. To assess the potential usefulness of tea for this purpose, we thought we should first know by in vitro analysis (i) how long period is required for the inactivation of SARS-CoV-2 by tea, and (ii) whether tea can inactivate the virus present in human saliva, before we start clinical studies to reveal (iii) whether tea ingestion/gargling inactivates the virus in human saliva in vivo, and (iv) how long period the viral inactivation effect in human saliva continues after tea ingestion/gargling.

In this study we tried to find answers to the above two questions (i) and (ii) in in vitro analyses. 
Results

We recently reported that treatment of SARS-CoV-2 with $500 \mu \mathrm{M}$ of TSA or TFDG for 1 min significantly reduced infectivity of the virus ${ }^{7}$. Then we treated the virus with $500 \mu \mathrm{M}$ TSA and TFDG for various periods, and determined the virus titer by $T_{C I D_{50}}$ assay (Fig. 1a). As results, $10 \mathrm{sec}$ was enough for TSA and TFDG to reduce virus infectivity to below the detectable limit (less than 1/1,000) (Fig. 1b).

We tested whether black and green tea inactivated SARS-CoV-2 in human saliva. Virus suspension was diluted in saliva from five different donors (purchased from Lee Biosolutions)(Supplemenatry Table S1), and treated with $1 \times$ black and green tea (vol:vol=1:1) for $10 \mathrm{sec}$ (Fig. 2a). After dilution, virus was infected into cells. It was clearly shown that both black and green tea significantly declined the titer of the virus in saliva (Fig. $2 b$ and $c)$.

To further confirm the virus inactivation caused by black tea treatment, virus in saliva was treated with black tea for $10 \mathrm{sec}$ and infected to cells. After culturing, secondary virus generated from the cells was estimated (Fig. 3a). As shown in Fig. 3b, virus titers in culture supernatants were either not detected or significantly lower compared with the titer of secondary virus released from the cells infected with intact virus. The results strongly suggest that the SARS-CoV-2 treated with black tea replicated at a significantly low degree, if any, in cells even in the presence of saliva during the treatment. 
Discussion

Saliva is the major origin of droplets that are released from SARS-CoV-2-infected persons and cause droplet infection, contact infection and aerosol infection to nearby persons ${ }^{6}$. Saliva contains various proteins including mucins, enzymes and immunoglobulins, nitrogenous products such as urea and ammonia, and electrolytes ${ }^{8}$. The present study shows that tea significantly inactivates SARS-CoV-2 even in the presence of saliva (Figs. 2-4).

In the experiments shown in Figs. 2 and 3, the virus was treated with tea for $10 \mathrm{sec}$, and the mixture was immediately diluted 1,000 -fold before an addition to the cells to allow viral attachment onto the cells. Tea diluted at 1,000-fold cannot effectively suppress virus infection as shown in our previous report ${ }^{7}$. Therefore, only $10 \mathrm{sec}$ was required for the inactivation of SARS-CoV-2 by tea.

Tea has a variety of biological effects including anti-viral activities, and tea catechins and their derivatives play important roles in these effects ${ }^{9-12}$. But these compounds are poorly absorbed in the intestine ${ }^{13,14}$, and their concentrations in plasma may not reach a sufficiently high level for inactivation of SARS-CoV-2. Therefore, tea may not be useful as a therapeutic medicine to treat respiratory and systemic manifestations of COVID-19 patients. Intake/gargling with tea by noninfected persons may be effective to some extent in preventing them from viral infection through the oral route, but may not be effective against viral entry via nasal and respiratory mucosa. But tea intake by SARS-CoV-2-infected persons could have greater significance as below.

It remains to be clarified to what extent SARS-CoV-2 in the saliva of SARS-CoV-2-infected persons can be inactivated in vivo by ingestion of, or gargling with, tea. Even if the virus in saliva is efficiently inactivated, the effect may be transient, because newly generated virus should reaccumulate in the saliva over time after the tea ingestion. Thus, it is important to reveal how long the anti-viral effect of tea will continue before virus reaccumulates in saliva to reach an enough high level to transmit infection to other persons through droplets. Turnover in vivo of the virus in saliva has not been reported so far, except for a study by Yoon et al. who examined a kinetic change of viral load in saliva in COVID-19 patients after mouthwash with chlorhexidine ${ }^{15}$. They reported that the viral load dropped 1 hour after the disinfectant mouthwash in two patients. The viral load remained low for 2 hours, and jumped up at 3 hours, suggesting reaccumulation of newly generated virus between 2 and 3 hours after transient elimination of preexisting virus by the mouthwash.

If ingestion/gargling with tea results in remarkable inactivation in vivo of SARS-CoV-2 in saliva and the effect lasts for some hours, tea may be applied to a novel "public health 
intervention"-like approach to prevent virus transmission among people. Because there are a considerable number of asymptomatic carriers, a lot of people including "healthy" people should join the activity during a period of explosive surge of COVID-19, and ingest or gargle with a small amount of tea before they have close contact with other persons, talk, sing, play sports and music, etc. for a couple of weeks, not only to protect themselves but also to protect their neighbors. Such a "mutual defense" campaign based on altruistic behavior may potentially contribute to attenuation of spread of virus infection within a population. Besides, tea ingestion/gargling may be recommended to patients with other diseases who undergo dental treatment, upper GI endoscopic examination, and so on, to prevent nosocomial infection of SARS-CoV-2.

But if the anti-viral effect in vivo of tea is poor and/or continues only for a very short period, usage of tea may be limited. Therefore, careful clinical studies are needed to determine the efficacy and longevity of SARS-CoV-2 inactivation by tea in saliva of humans in vivo.

Finally, it should be noted that, although tea has been ordinary consumed by a large number of people for many years, intake of an excess amount of tea could cause adverse events including liver toxicity ${ }^{16,17}$. 
Materials and Methods

Cells, virus, and culture medium

VeroE6/TMPRSS2 cells ${ }^{18}$ were obtained from Japanese Collection of Research Biosources Cell Bank, National Institute of Biomedical Innovation (Osaka, Japan) and cultured in Dulbecco's modified Eagle's minimum essential medium (DMEM) (Nissui Pharmaceutical Co. Ltd., Tokyo, Japan) supplemented with G418 disulfate $(1 \mathrm{mg} / \mathrm{mL})$, penicillin (100 units $/ \mathrm{mL})$, streptomycin $(100 \mu \mathrm{g} / \mathrm{mL}), 5 \%$ fetal bovine serum at $37^{\circ} \mathrm{C}$ in a $5 \% \mathrm{CO}_{2} / 95 \%$ in a humidified atmosphere. SARS-CoV-2 (Japan/AI/I-004/2020) were kindly provided from Japan National Institute of Infectious Diseases (Tokyo, Japan) and propagated using VeroE6/TMPRSS2 cells.

Tea, chemicals and saliva

To prepare freeze-dried powders of tea extract, tea extracts were prepared by soaking $40 \mathrm{~g}$ of ground up and homogenized tea leaves in $2,000 \mathrm{~mL}$ water at $80^{\circ} \mathrm{C}$ for $30 \mathrm{~min}$. After centrifugation at 4,000 rpm for $15 \mathrm{~min}$, supernatants were collected and filtrated through Toyo No. 2 filter papers, followed by evaporation and freeze-drying. EGCG and TFDG were purchased from FUJIFILM Wako Pure Chemical Corporation (Osaka, Japan). TSA was synthesized from EGCG and purified as described. ${ }^{7,19}$ Human saliva was purchased from Lee Biosolutions (Maryland Heights, MO, USA) (Supplementary Table 1).

$\mathrm{TCID}_{50}$ assay for virus treated with TFDG and TSA

Solutions of TSA and TFDG were diluted in MS (DMEM supplemented with $0.5 \%$ FBS) to a concentration of $2 \mathrm{mM}$. Twenty $\mu \mathrm{L}$ of $\mathrm{x} 2$ serum-free DMEM was added to $20 \mu \mathrm{L}$ of each solution, and $40 \mu \mathrm{L}$ of SARS-CoV-2 suspension $\left(2 \times 10^{6} \mathrm{TCID}_{50} / 50 \mu \mathrm{L}\right)$ was added, followed by incubation at room temperature for $10,20,30$ or $60 \mathrm{sec}$. Immediately, the mixture was diluted 1,000-fold in serum-free DMEM, followed by a serial dilution at 10-fold with MS in triplicate in 96-well-plates. Chilled on ice, $100 \mu \mathrm{L}$ of each sample was added to the VeroE6/TMPRSS2 cells that had been seeded in 96-well-plates at $5 \times 10^{4} / 100 \mu \mathrm{L} /$ well a day before. After incubation for 1 hour to allow attachment of the virus onto the cells, supernatant was replaced by fresh culture medium, and cells were cultured for 4 days. Cells were then washed, fixed and stained with crystal violet solution to estimate CPE as described ${ }^{20}$. TCID $_{50}$ values were calculated by Reed-Muench method.

$\mathrm{TCID}_{50}$ assay for virus in saliva treated with tea

Freeze-dried powders of green and black tea were dissolved in sterilized distilled water at 
$78^{\circ} \mathrm{C}$ for $10 \mathrm{~min}$ to prepare $\mathrm{x} 1$ concentration of original tea. After chilling at room temperature, each tea was passed through a $0.45 \mu \mathrm{m}$ filter. Human saliva was sterilized by UV irradiation for $30 \mathrm{~min}$. Virus suspension $\left(3.0 \times 10^{5} \mathrm{TCID}_{50} / 5 \mu \mathrm{L}\right)$ was mixed with $45 \mu \mathrm{L}$ of saliva or water, followed by an addition of tea at 1:1 (vol:vol) for $10 \mathrm{sec}$. Immediately, the mixture was diluted 1,000-fold in serum-free DMEM, followed by a serial dilution at 10 -fold with MS and $\mathrm{TCID}_{50}$ assay as above.

$\mathrm{TCID}_{50}$ assay for secondary virus

Virus suspension in saliva was treated with black tea or distilled water for $10 \mathrm{sec}$, and immediately diluted 1,000 -fold as above. One-hundred $\mu \mathrm{L}$ of the mixture was added to the cells that had been seeded in 24-well-plates at $2.5 \times 10^{5} /$ well a day before. After incubation for 1 hour to allow attachment of the virus onto the cells, supernatant was replaced by 500 $\mu \mathrm{L}$ of fresh culture medium, and cells were culture for 10 hours. An aliquot of culture supernatant was subjected to a serial dilution at 10 -fold with $M S$ and $T_{C I D}$ assay as above.

Statistical analysis

Statistical significance was analyzed by Student's $t$ test, and $\mathrm{P}<0.05$ was considered significant.

$\mathrm{COI}$

This study was partially funded by ITO EN, Itd, Tokyo, Japan. The company also provided tea samples, sample preparations and discussion with authors, but did not involve in the design of the study, collection and analyses of data, interpretation of results, preparation of the manuscript, or the decision to publish the results. 


\section{References}

1 Patel, K. P. et al. Transmission of SARS-CoV-2: an update of current literature. Eur J Clin Microbiol Infect Dis 39, 2005-2011, doi:10. 1007/s10096-020-03961-1 (2020).

2 Khan, S., Liu, J. \& Xue, M. Transmission of SARS-CoV-2, Required Developments in Research and Associated Public Health Concerns. Front Med (Lausanne) 7, 310, doi:10. 3389/fmed, 2020.00310 (2020).

3 Li, Y. et al. Saliva is a non-negligible factor in the spread of COVID-19. Mol Oral Microbiol 35, 141-145, doi:10.1111/omi. 12289 (2020).

4 Peng, X. et al. Transmission routes of 2019-nCoV and controls in dental practice. Int J Oral Sci 12, 9, doi:10. 1038/s41368-020-0075-9 (2020).

$5 \mathrm{Xu}, \mathrm{R}$. et al. Saliva: potential diagnostic value and transmission of 2019-nCoV. Int J Oral Sci 12, 11, doi:10. 1038/s41368-020-0080-z (2020).

6 Shamsoddin, E. Saliva: a diagnostic option and a transmission route for 2019-nCoV. Evid Based Dent 21, 68-70, doi:10. 1038/s41432-020-0104-8 (2020).

7 Ohgitani, E. et al. Significant inactivation of SARS-CoV-2 by a green tea catechin, a catechin-derivative and galloylated theaflavins in vitro. BioRxiv, doi:10. 1101/2020.12.04.412098 (2020).

8 Humphrey, S. P. \& Williamson, R. T. A review of saliva: normal composition, flow, and function. J Prosthet Dent 85, 162-169, doi:10. 1067/mpr. 2001. 113778 (2001).

9 Khan, N. \& Mukhtar, H. Tea Polyphenols in Promotion of Human Health. Nutrients 11, doi:10. 3390/nu11010039 (2018).

10 Ohishi, T., Goto, S., Monira, P., Isemura, M. \& Nakamura, Y. Anti-inflammatory Action of Green Tea. Antiinflamm Antiallergy Agents Med Chem 15, 74-90, doi:10. 2174/1871523015666160915154443 (2016).

11 Singh, B. N., Shankar, S. \& Srivastava, R. K. Green tea catechin, epigallocatechin-3-gallate (EGCG): mechanisms, perspectives and clinical applications. Biochem Pharmacol 82, 1807-1821, doi:10. 1016/j. bcp. 2011.07.093 (2011).

12 Xu, J., Xu, Z. \& Zheng, W. A Review of the Antiviral Role of Green Tea Catechins. Molecules 22, doi:10.3390/molecules22081337 (2017).

13 Scholl, C. et al. Population nutrikinetics of green tea extract. PLoS One 13, e0193074, doi:10. 1371/journal, pone. 0193074 (2018).

14 Lee, M. J. et al. Pharmacokinetics of tea catechins after ingestion of green tea 
and (-)-epigallocatechin-3-gallate by humans: formation of different metabolites and individual variability. Cancer Epidemiol Biomarkers Prev 11, 1025-1032 (2002).

15 Yoon, J. G. et al. Clinical Significance of a High SARS-CoV-2 Viral Load in the Saliva. J Korean Med Sci 35, e195, doi:10. 3346/jkms. 2020. 35. e195 (2020).

16 Additives, E. P. o. F. et al. Scientific opinion on the safety of green tea catechins. EFSA J 16, e05239, doi:10.2903/j. efsa. 2018. 5239 (2018).

17 Hu, J., Webster, D., Cao, J. \& Shao, A. The safety of green tea and green tea extract consumption in adults - Results of a systematic review. Regul Toxicol Pharmacol 95, 412-433, doi:10. 1016/j. yrtph. 2018.03.019 (2018).

18 Matsuyama, S. et al. Enhanced isolation of SARS-CoV-2 by TMPRSS2-expressing cells. Proc Nat1 Acad Sci USA 117, 7001-7003, doi:10. 1073/pnas. 2002589117 (2020).

19 Shii, T., Miyamoto, M., Matsuo, Y., Tanaka, T. \& Kouno, I. Biomimetic one-pot preparation of a black tea polyphenol theasinensin A from epigallocatechin gallate by treatment with copper(II) chloride and ascorbic acid. Chem Pharm Bull (Tokyo) 59, 1183-1185, doi:10.1248/cpb. 59. 1183 (2011).

20 Pezzotti, G. et al. Instantaneous "catch-and-ki11" inactivation of SARS-CoV-2 by nitride ceramics. Clin Trans1 Med 10, e212, doi:10.1002/ctm2. 212 (2020). 
Legends to the Figures

Fig. 1 TSA and TFDG inactivated SARS-CoV-2 within 10 sec.

SARS-CoV-2 was treated with $500 \mu \mathrm{M}$ of TSA or TFDG for the indicated periods, immediately followed by a 1,000 -fold dilution with $M S$. TCID 50 assay was performed as described in the Materials and Methods. Scheme of experiment (a) and virus titer of each sample (means \pm S.D., $N=3$ ) (b) are shown.

Fig. 2 SARS-CoV-2 was inactivated by tea even in the presence of saliva.

SARS-CoV-2 was diluted in saliva from five independent donors or in distilled water (DW) as a control (a). Black tea, green tea or DW was added to the virus/saliva for $10 \mathrm{sec}$, immediately followed by a 1,000 -fold dilution with MS. TCID 50 assay was performed as described in Fig. 1. Scheme of experiment (a) and virus titer of each sample (means \pm S.D., $\mathrm{N}=3)(\mathrm{b})$ are shown.

Fig. 3 Treatment with tea significantly inhibited reproduction of SARS-CoV-2 in cells SARS-CoV-2 was diluted in saliva from five independent donors or in DW. Black tea or DW was added to the virus/saliva for $10 \mathrm{sec}$, immediately followed by a 1,000-fold dilution with MS. After infection, cells were cultured for $10 \mathrm{~h}$, and culture supernatants were subjected to $\mathrm{TCID}_{50}$ assay as in Fig. 1. Scheme of experiment (a) and virus titer of each sample (means \pm S.D., $N=3$ )(b) are shown. ${ }^{*} \mathrm{P}<0.05$ vs. DW. 


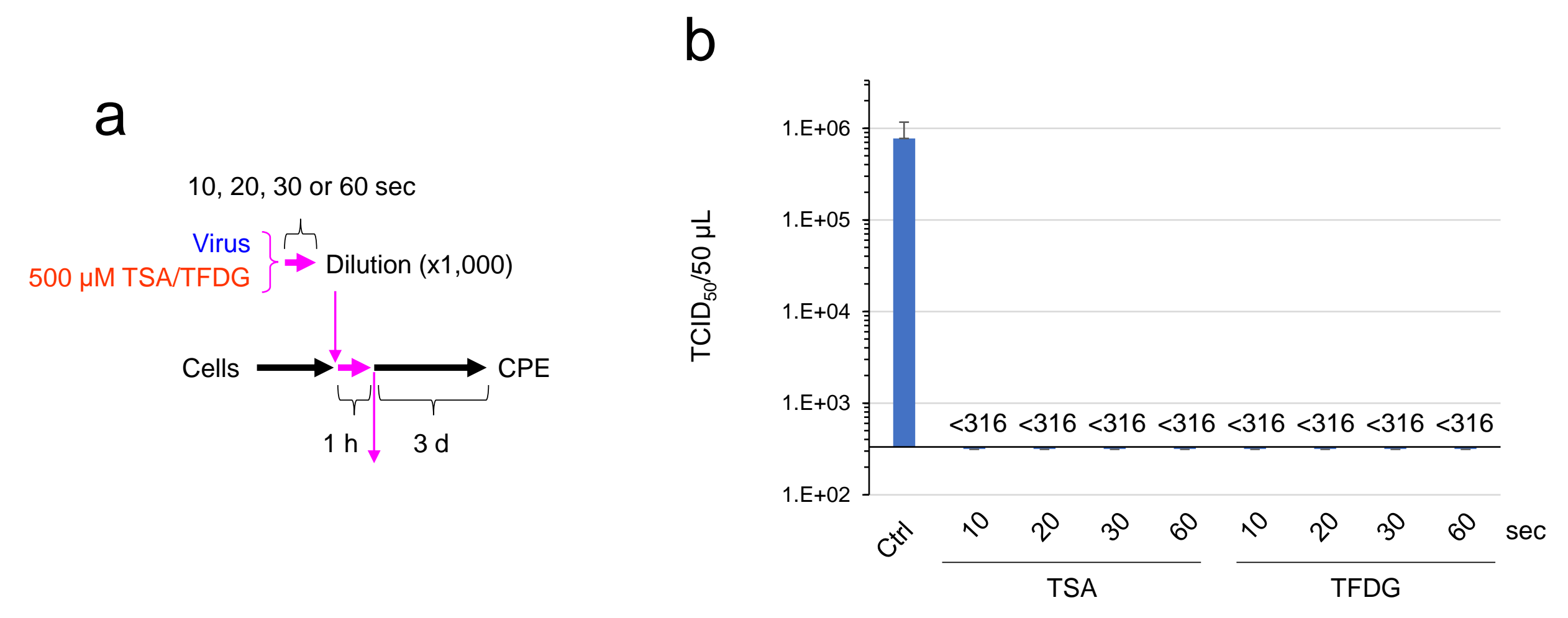


b

a

$10 \mathrm{sec}$

Virus in saliva or in DW Black tea, Green tea or DW $\} \Rightarrow$ Dilution $(x 1,000)$

Cells

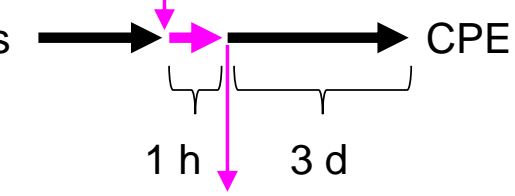

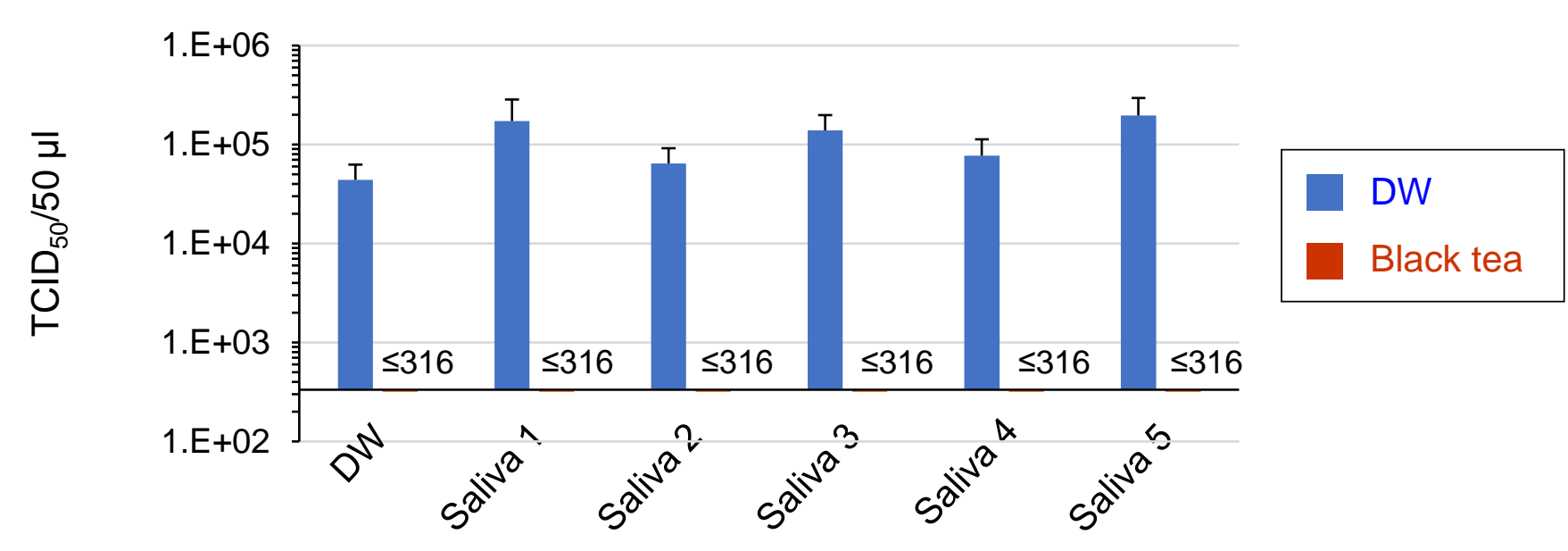

C

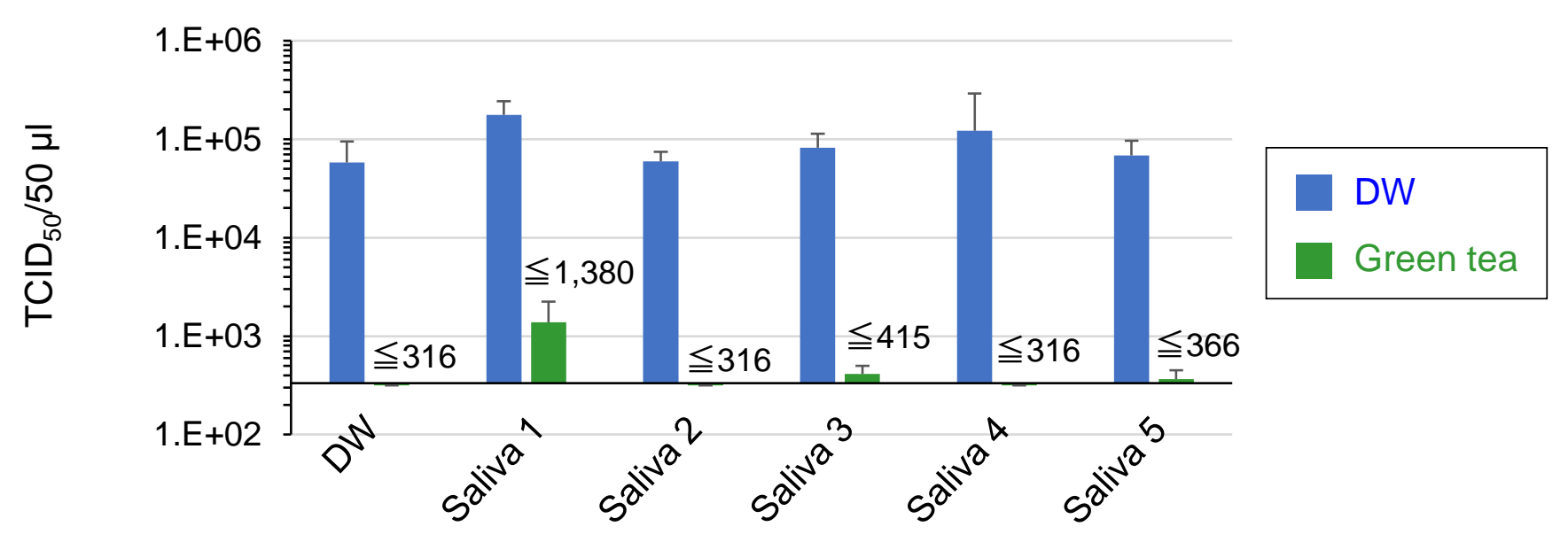


a

$10 \mathrm{sec}$

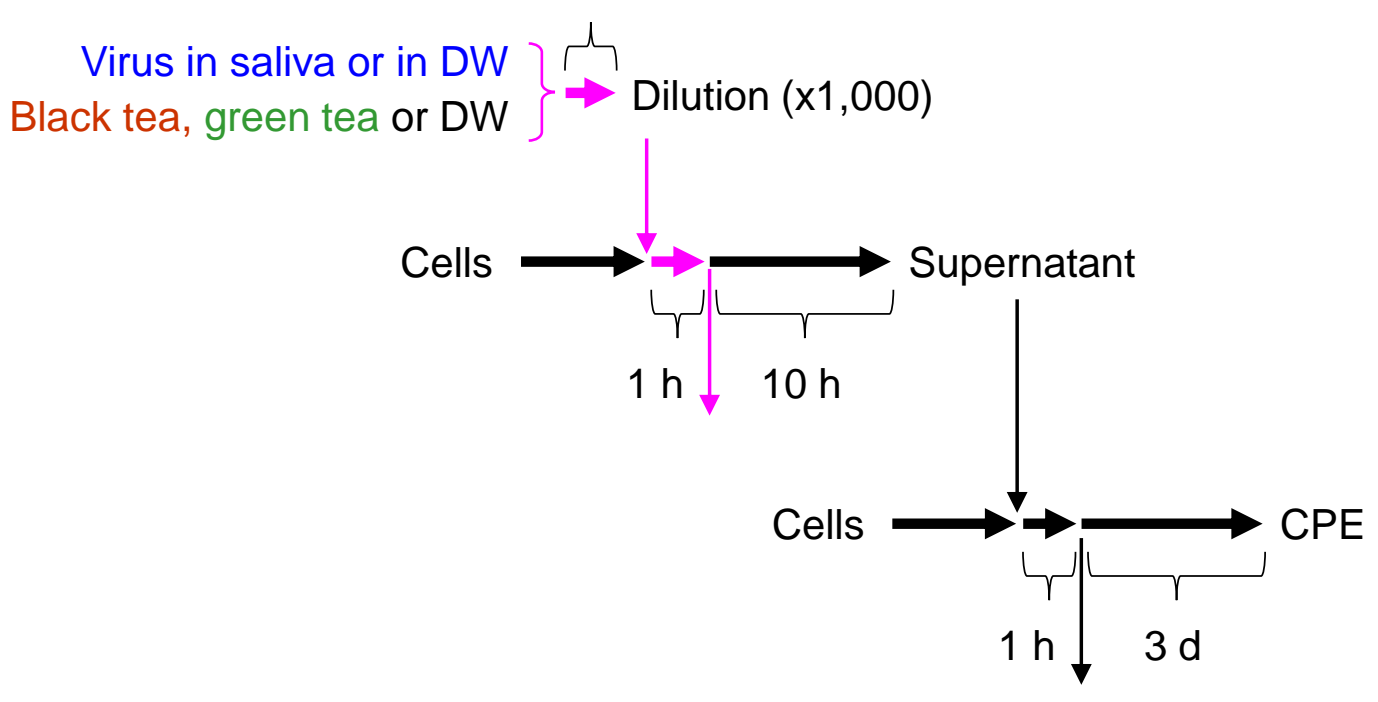

b

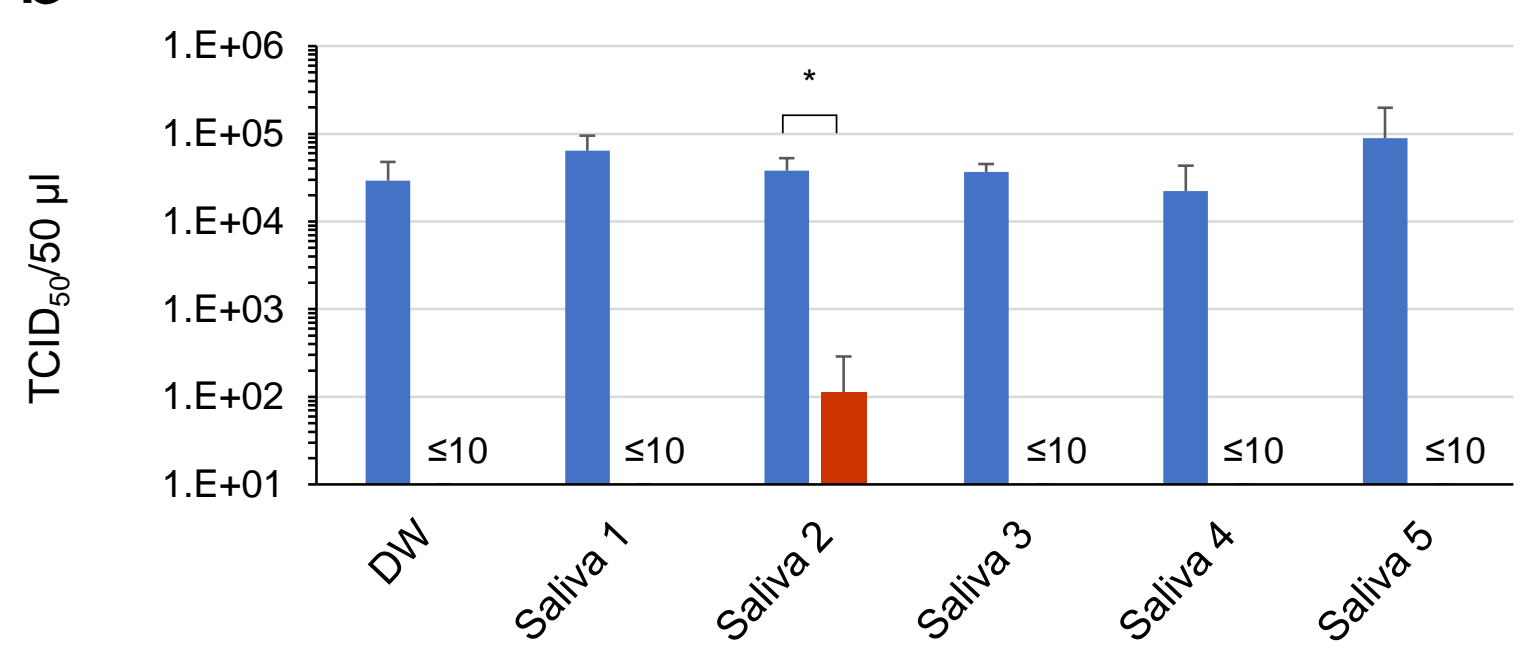

DW

Black tea 


\begin{tabular}{|l|l|l|l|}
\hline Number & Birth year & Gender & Race \\
\hline 1 & 1984 & Male & Caucasian \\
\hline 2 & 1989 & Male & African American \\
\hline 3 & 1986 & Male & Caucasian \\
\hline 4 & 1967 & Female & Caucasian \\
\hline 5 & 1968 & Male & Caucasian \\
\hline
\end{tabular}

Supplementary Table S1

Donors of saliva that was purchased from Lee Biosolutions (Maryland Heights, MO, USA) and used in the study are shown. 